\title{
A back-to-front derivation: the equal spacing of quantum levels is a proof of simple harmonic oscillator physics
}

\author{
David L Andrews and Luciana C Dávila Romero \\ Nanostructures and Photomolecular Systems, School of Chemical Sciences, University of East \\ Anglia, Norwich NR4 7TJ, UK \\ E-mail: david.andrews@physics.org
}

Received 23 April 2009, in final form 21 July 2009

Published 5 October 2009

Online at stacks.iop.org/EJP/30/1371

\begin{abstract}
The dynamical behaviour of simple harmonic motion can be found in numerous natural phenomena. Within the quantum realm of atomic, molecular and optical systems, two main features are associated with harmonic oscillations: a finite ground-state energy and equally spaced quantum energy levels. Here it is shown that there is in fact a one-to-one mapping between the provision of equally spaced quantum energy levels and simple harmonic motion. The analysis establishes that the Hamiltonian of any system featuring quantized energy levels in an evenly spaced, infinite set must have a quadratic dependence on a pair of canonically conjugate variables. Moreover, specific physical inferences can be drawn. For example, exploiting this 'back-to-front' derivation, and based on the known existence of photons, it can be proved that an electromagnetic energy density is quadratic in both the electric and magnetic fields.
\end{abstract}

\section{Introduction}

The dynamics of simple harmonic oscillation is pervasive across the whole of physics. In the atomic, molecular and optical realm where quantum features become apparent a host of applications can be found, extending from the vibrations of nuclei within molecules to quantum optical states of light $[1,2]$. Two of the key, most familiar features of the quantum harmonic oscillator are its finite ground-state energy and the equidistant spacing between a limitless succession of quantum energy levels [3-5]. In the context of a quantum world, the former is not especially remarkable, even though it has significant implications; it is in many respects an obvious corollary of the uncertainty principle. However, the constant energy spacing, within a set of infinite extent, has even more extensive consequences, and in the case of an irreducible system it is unique to the simple harmonic oscillator (SHO). As is well known, this is one of the most striking features to emerge from detailed solutions of the Schrödinger 
equation. Remarkably, it is a feature that persists in a more complex system formed by a set of one-dimensional oscillators coupled with an inverse squared potential, known as the Calogero-Sutherland model [6-9]. It has been shown that an exact solution to the latter system is equivalent to a set of uncoupled harmonic oscillators with matching eigenvalues, apart from a shift of the ground-state energy; however, in such a case, the particles are spatially confined $[10,11]$. This paper focuses upon systems that are irreducible, in the sense of relating to just one degree of freedom, and where there are no restrictions on the motion-as is usual for simple harmonic oscillation.

In the following, it is our aim to address the question: Is it possible to derive the dynamical details that characterize unconfined SHO dynamics in real space, based only on a recognition of quantized energy levels forming an evenly spaced, infinite set? We shall show that it does indeed prove possible; there is a one-to-one mapping between the provision of equally spaced quantum energy levels and simple harmonic motion. The derivation is argued pedagogically, and in the pursuit of a relentless logic it exploits quantum principles rather differently to the more usual progression that begins with the construction of a system Hamiltonian-see for example reference [12]. In contrast, the presented derivation culminates in that Hamiltonian from which the quadratic dependence on a pair of canonically conjugate variables such as position and momentum can be readily established. Moreover, specific physical inferences can be drawn. For example, exploiting this 'back-to-front' derivation and based on the known existence of photons, it can be proven (rather than stated or derived from Maxwell's equations) that an electromagnetic energy density has to be quadratic in both electric and magnetic fields [13].

The analysis that follows is suitable, and should prove enlightening, for those who are relatively new to quantum mechanics—only a basic familiarity with operators and Dirac notation [14] are assumed. As well as furnishing physical insights, a number of important quantum principles are also introduced and their implementation illustrated in the course of the following development.

\section{Derivation}

The analysis is to be founded upon a single fact that observations on a certain physical system reveal an unbounded set of energy levels that are equally spaced, and which can therefore be indexed by an infinite series of positive integers $n$. In Dirac notation, the quantum states can be represented by kets $|n\rangle$, and since these must be eigenstates of the Schrödinger equation we can write

$$
H|n\rangle=E_{n}|n\rangle .
$$

Here, $E_{n}$ is the energy associated with the state $|n\rangle$; for simplicity, all such states are assumed to be non-degenerate and their wavefunctions normalized. Any system must have a state of lowest energy; this state we can arbitrarily designate as $|0\rangle$, with $E_{0}$ signifying that energy. We cannot assume the ground state to have zero energy; the value of $E_{0}$ is therefore to be determined. The one measurable quantity that can be introduced is the spacing between adjacent energy levels which, since it is a quantum feature, can be written arbitrarily as $\hbar \omega$, but without laying any claim to any deeper significance for the value of the frequency unit $\omega$. (Obviously, the physical significance of this frequency will be determined by the properties of the system that gives rise to this quantum behaviour.) Accordingly, $E_{n}$ can be expressed as

$$
E_{n}=E_{0}+n \hbar \omega .
$$




\subsection{Ladder operations}

To begin the analysis, it will prove expedient to introduce two operators, $a$ and $a^{\dagger}$, assumed to be independent of $n$, whose properties are defined by

$$
\begin{aligned}
& a|n\rangle=c_{n}|n-1\rangle, \\
& a^{\dagger}|n\rangle=d_{n}|n+1\rangle .
\end{aligned}
$$

By way of justification, it will be shown in the following that these relations self-consistently, and without further assumptions, lead to the required energy level structure given by equation (2) — as will emerge in (18) below. Interpreting equations (3) and (4), the lowering operator, $a$, operates upon a state to deliver the state one below it on the ladder of quantum levels; the raising operator, $a^{\dagger}$, operates on a state to deliver the one above. The coefficients $c_{n}$ and $d_{n}$ are numerical and may be complex. Since $a^{\dagger}$ effects the time-reverse operation to $a$, it is the latter's Hermitian conjugate [15]. In terms of quantity calculus, the two operators may be chosen to be dimensionless. However, both the operator on the left and the coefficient on the right in each equations (3) and (4) are currently undetermined, and each is arbitrarily scalable until further conditions are applied. For example, for any scalar $\lambda$, we could write $a^{\prime}=\lambda a$ and $c_{n}^{\prime}=\lambda c_{n}$, and the primed quantities would still satisfy equation (3).

Equations (3) and (4) also introduce as yet unknown numerical coefficients on the right, their subscript indices indicating that the coefficient values are allowed to depend on the number $n$. In the lowering operator case, it is easy to show that there must be some dependence of $c_{n}$ on $n$-in other words, the coefficient cannot have the same value for all $n$. To see this, consider the implementation of (3) on the only state we have specific knowledge about, i.e. the ground state. Unless $c_{0}=0$, equation (3) delivers a clearly meaningless result, suggesting that we can obtain a state lower in energy than the one already defined as having the lowest attainable energy, the ground state. Incontrovertibly, we must therefore have

$$
a|0\rangle=0 \text {. }
$$

Equally clearly we cannot have $c_{n}=0$ for all $n$, or else $a$ would be a null operator, so the coefficient values must in general depend on $n$.

Next, we can unveil a simple connection between the $c_{n}$ and $d_{n}$ coefficients. This is done by completing and evaluating Dirac brackets, using (3) and (4), as follows:

$$
\begin{aligned}
& \langle n-1|a| n\rangle=\left\langle n-1\left|c_{n}\right| n-1\right\rangle=c_{n} \\
& \Rightarrow c_{n}^{*}=\left\langle n\left|a^{\dagger}\right| n-1\right\rangle=\left\langle n\left|d_{n-1}\right| n\right\rangle=d_{n-1} \\
& \therefore d_{n}=c_{n+1}^{*},
\end{aligned}
$$

where the asterisk denotes a complex conjugate. In the second line above, use of the general Dirac relationship $\langle r|\hat{O}| s\rangle=\left\langle s\left|\hat{O}^{\dagger}\right| r\right\rangle^{*}$ for an operator $\hat{O}$ and its Hermitian conjugate $\hat{O}^{\dagger}$ [12] has been made. Using (6), equation (4) can thus be rewritten as

$$
a^{\dagger}|n\rangle=c_{n+1}^{*}|n+1\rangle \text {. }
$$

Having introduced two operators, it is logical to investigate their commutation relationship. Consider the results of applying the operator products $a^{\dagger} a$ and $a a^{\dagger}$ to the states $|n\rangle$; from (3) and (7) we have

$$
\begin{aligned}
& a^{\dagger} a|n\rangle=c_{n} a^{\dagger}|n-1\rangle=c_{n} c_{n}^{*}|n\rangle, \\
& a a^{\dagger}|n\rangle=c_{n+1}^{*} a|n+1\rangle=c_{n+1} c_{n+1}^{*}|n\rangle
\end{aligned}
$$


Equations (8) and (9) serve to establish that the states $|n\rangle$ are eigenstates of both $a^{\dagger} a$ and $a a^{\dagger}$, but the corresponding eigenvalues $\left|c_{n}\right|^{2}$ and $\left|c_{n+1}\right|^{2}$ are different. From these two equations, we can deduce that

$$
\left\{\left(a a^{\dagger}-a^{\dagger} a\right)-\left(\left|c_{n+1}\right|^{2}-\left|c_{n}\right|^{2}\right)\right\}|n\rangle=0 .
$$

Since this is valid for all states $|n\rangle$, we can deduce that

$$
\left[a, a^{\dagger}\right]=\left|c_{n+1}\right|^{2}-\left|c_{n}\right|^{2}, \quad \forall n
$$

with the usual meaning of the commutator [14], i.e. $\left[a, a^{\dagger}\right] \equiv a a^{\dagger}-a^{\dagger} a$. The left-hand side of equation (11) is equal to a scalar, say $\eta$, clearly independent of $n$. Therefore, using $\left|c_{n+1}\right|^{2}-\left|c_{n}\right|^{2}=\eta$, it is possible to find an expression for $\left|c_{n}\right|^{2}$ :

$$
\begin{aligned}
& \left|c_{0}\right|^{2}=0 \\
& \left|c_{1}\right|^{2}=\eta+\left|c_{0}\right|^{2}=\eta \\
& \left|c_{2}\right|^{2}=1+\left|c_{1}\right|^{2}=1+\eta \\
& \vdots \\
& \left|c_{n+1}\right|^{2}=1+\left|c_{n}\right|^{2}=n+\eta,
\end{aligned}
$$

where the known value of $c_{0}=0$ has been used. The explicit use of an iterative procedure at this juncture exploits the absence of an upper bound on the observed, equally spaced set of energy levels; the significance is that this is a feature setting the physics apart from that of some other kinds of system-such as the Zeeman-split sub-levels of angular momentum states $[5,16]$.

To continue, as mentioned earlier, both the operators $a$ and $a^{\dagger}$ and the coefficients $c_{n}$ are currently incompletely determined, in the sense that each is arbitrarily scalable by any constant that is independent of $n$. This freedom invites the simple choice $\eta=1$, effecting a removal of such a scaling ambiguity. The commutation relation between the annihilation and the creation operator is thus

$$
\left[a, a^{\dagger}\right]=1
$$

and the general form for the $c_{n}$ coefficient is

$$
c_{n}=\sqrt{n}
$$

Although there is a more general solution $c_{n}=\mathrm{e}^{\mathrm{i} \phi} \sqrt{n}$ that carries a phase factor, the latter has no physical significance and can be set to unity without loss of generality.

To recap, from equations (3), (4), (6) and (14), we have ascertained that the ladder operators work as follows:

$$
\begin{aligned}
& a|n\rangle=\sqrt{n}|n\rangle, \\
& a^{\dagger}|n\rangle=\sqrt{n+1}|n+1\rangle .
\end{aligned}
$$

We also have

$$
a^{\dagger} a|n\rangle=n|n\rangle
$$

which establishes $a^{\dagger} a$ as the number operator. 


\subsection{Hamiltonian deconstruction}

From the foundational equation (2), it is now evident from equations (1) and (17) that we can write the Hamiltonian operator as

$$
H=a^{\dagger} a \hbar \omega+E_{0} .
$$

While aiming to retaining generality, it remains only to rework this operator so as to interpret the dynamical form upon which it must be based. To this end, it is worth recalling the usual starting point for solving the Schrödinger equation, a classical energy expression promoted to the quantum operator form. The operators so formed must be Hermitian, i.e. they must behave as any operator $\hat{A}$ that satisfies the relation $\langle\phi|\hat{A}| \psi\rangle=\langle\psi|\hat{A}| \phi\rangle^{*}$, since this is a property of all operators that represent physical observables [15]. Recognition of this provides a guiding principle that the physics will be most clearly exhibited if $H$ is written in terms of Hermitian operators. Since the two operators that feature in (18) form a Hermitian conjugate pair, they clearly need not be Hermitian (self-adjoint).

To proceed, we observe that it is always possible to write any operator $\hat{O}$ as a sum of operators in the form

$$
\hat{O}=\hat{O}_{1}+\mathrm{i} \hat{O}_{2}
$$

where $\hat{O}_{1}$ and $\hat{O}_{2}$ are both Hermitian [15]. The proof is straightforward: let $\hat{O}^{\dagger}$ be the Hermitian conjugate of $\hat{O}$; thus,

$$
\begin{aligned}
\langle\phi|\hat{O}| \psi\rangle & =\left\langle\phi\left|\hat{O}_{1}\right| \psi\right\rangle+\mathrm{i}\left\langle\phi\left|\hat{O}_{2}\right| \psi\right\rangle \\
& =\left\langle\psi\left|\hat{O}_{1}\right| \phi\right\rangle^{*}+\mathrm{i}\left\langle\psi\left|\hat{O}_{2}\right| \phi\right\rangle^{*} \\
& =\left\langle\psi\left|\hat{O}_{1}-\mathrm{i} \hat{O}_{2}\right| \phi\right\rangle^{*}=\left\langle\psi\left|\hat{O}^{\dagger}\right| \phi\right\rangle^{*}
\end{aligned}
$$

In the second line, use of the Hermiticity of $\hat{O}_{1}$ and $\hat{O}_{2}$ has been made, and the result of the third line establishes the Hermitian conjugacy of $\hat{O}$ and $\hat{O}^{\dagger}$. Applying this result, we can choose to express both $a$ and $a^{\dagger}$ in terms of two Hermitian operators $P$ and $Q$, still both dimensionless:

$$
\begin{aligned}
& a=P-\mathrm{i} Q, \\
& a^{\dagger}=P+\mathrm{i} Q .
\end{aligned}
$$

Hence, since from (13) we have $\left[a, a^{\dagger}\right]=1$, it follows that

$$
\begin{aligned}
& (P-\mathrm{i} Q)(P+\mathrm{i} Q)-(P+\mathrm{i} Q)(P-\mathrm{i} Q)=2 \mathrm{i} P Q-2 \mathrm{i} Q P=2 \mathrm{i}[P, Q] . \\
& \therefore[P, Q]=-\frac{\mathrm{i}}{2} .
\end{aligned}
$$

Equation (22) establishes the operators $P$ and $Q$ as canonically conjugate $[14,17]$, standing in the same kind of relationship as a momentum and position coordinates along a common axis. This is also consistent with their joint engagement in an uncertainty relation of the form $\Delta Q \Delta P \geqslant \hbar / 2$, where $\Delta X=\left(\left\langle X^{2}\right\rangle-\langle X\rangle^{2}\right)^{1 / 2}[18,19]$, signifying a reciprocal interdependence in the minimum certainties of measurement for the physical properties associated with each operator.

Using the above result (22), we can rewrite the number operator in terms of $P$ and $Q$ as follows:

$$
a^{\dagger} a=(P+\mathrm{i} Q)(P-\mathrm{i} Q)=P^{2}+Q^{2}-\mathrm{i}[P, Q]=P^{2}+Q^{2}-\frac{1}{2},
$$

and hence, from (18),

$$
H=\left(P^{2}+Q^{2}\right) \hbar \omega+E_{0}-\frac{1}{2} \hbar \omega .
$$


One last, general conclusion can be drawn, by considering the Hamiltonian (24) as a nexus of quantum and classical physics. Standard procedures in quantum mechanics will produce any quantum Hamiltonian from a counterpart expression for the classical energy (expressed as a sum of kinetic and potential energy terms), through the promotion of dynamical variables to operator form. Here, the operators $P$ and $Q$ will originate in dynamical variables that, in contrast to the quantum behaviour, can simultaneously take zero values. Moreover, when the left-hand side of (24) is interpreted as a classical energy, it is certainly permissible for the result then to be zero. Consequently it must be concluded that

$$
E_{0}=\frac{1}{2} \hbar \omega
$$

therefore establishing a value for the previously undetermined ground-state energy of the quantum system. Hence, it emerges that the Hamiltonian is expressible in two equivalent forms:

$$
H=\left(a^{\dagger} a+\frac{1}{2}\right) \hbar \omega=\left(P^{2}+Q^{2}\right) \hbar \omega .
$$

It is notable that the ground-state energy $\frac{1}{2} \hbar \omega$ which appears explicitly in the ladder operator form in (25) is not separable in the $P^{2}+Q^{2}$ formula.

To summarize the above development: it has been shown that any infinite set of equally spaced discrete energy levels owes its origins to a Hamiltonian that accommodates terms quadratic in a pair of dynamical variables corresponding to non-commuting quantum operators-specifically, a pair whose commutator is a constant. Since this is the only general constraint upon $P$ and $Q$, it is obvious that the associations of each operator individually are interchangeable, to within a phase factor. Indeed there is more than this ambiguity, for it is easy (and an instructive class exercise) to show that both the Hamiltonian (26) and the commutation relationship are invariant under any unitary transformation into new operators $P^{\prime}$ and $Q^{\prime}$ defined by

$$
P^{\prime}=P \cos \theta+Q \sin \theta ; \quad Q^{\prime}=-P \sin \theta+Q \cos \theta .
$$

Since the Hamiltonian has dimensions of energy, it necessarily has even parity in both time and space dimensions. As $Q$ and $P$ both appear quadratically in (26), each can have either odd or even parity, in each dimensional respect. To go any further now-and to discover a physical significance for the frequency $\omega$-requires a focus on a specific system.

\section{Real systems}

A host of quantum systems display dynamical behaviour of simple harmonic nature, and it is not our purpose to exhibit the relevance of the above analysis to each of them. For illustrative purposes, and to exhibit the breadth of application, we simply focus on two physically very different cases. The first is obvious and familiar-the case of mechanical oscillation; we then turn to the core equations of quantum optics, where the analysis lends additional insights to issues surrounding the quantization of electromagnetic radiation.

\subsection{Mechanical oscillation}

In classical mechanics, an $\mathrm{SHO}$ is a closed system whose energy, as expressed for a particle with position $q$ and momentum $p$, is given by

$$
E=\frac{p^{2}}{2 m}+\frac{1}{2} k q^{2}
$$

Here $m$ is the particle's point mass and $k$ the elastic constant of the system [20]. Away from equilibrium the restoring force, $-k q$, is proportional to displacement in accordance 
with Hooke's law. More generally, the SHO affords a first approximation to any system displaced by a small amount from a stable equilibrium position $q_{0}$. A particle moving in this vicinity is subjected to a potential $V(q)$ that can be approximated by a Taylor expansion [20], in which case the leading term $V\left(q_{o}\right)$ represents an arbitrary origin on the energy scale, and $\mathrm{d}^{2} V /\left.\mathrm{d} q^{2}\right|_{q=q_{o}}$ plays the role of the elastic constant $k$ : there is no term linear in the displacement because its multiplier disappears through the equilibrium condition on the force, $\mathrm{d} V /\left.\mathrm{d} q\right|_{q=q_{o}}=0$. Solving the dynamics of the mechanical system yields the general solution $q(t)=A \cos (\sqrt{k / m t+\phi})$, in which the amplitude $A$ and phase $\phi$ are determined by initial conditions, i.e. $q(0)$ and $\dot{q}(0)$. From the periodicity of the cosine function, it follows that the circular frequency characterizing the system is $\sqrt{\mathrm{k} / \mathrm{m}}$.

Since the generic quantum Hamiltonian (26) can always be secured by promoting the classical energy expression for a particular system into the operator status, it is now possible to identify a more specific form for the $P$ and $Q$ operators in the case of mechanical oscillation. The linear momentum and position variables $p$ and $q$ in (27) become operators $\hat{p}$ and $\hat{q}$, which transform into dimensionless form as follows:

$$
\left.\begin{array}{l}
p \rightarrow \hat{p}=\sqrt{2 m \hbar \omega} P \\
q \rightarrow \hat{q}=\sqrt{\frac{2 \hbar}{m \omega}} Q
\end{array}\right\} .
$$

In principle, the quantities on the right in (28) are interchangeable, but as written the $P$ operator will have odd parity in time - as befits its representation of momentum. In the spirit of the present analysis, the conclusion that can be drawn from making the identification (28) is that the frequency unit, $\omega$, introduced at the beginning of section 2 , is none other than the circular frequency of classical oscillation, $\sqrt{k / m}$. This confers clear physical meanings on the energy unit quantifying the spacing between quantum energy levels, as in equation (2), and also the zero-point energy $E_{0}$, given by (25). This completes a deduction of simple harmonic motion from the observation of an infinite set of equally spaced quantum energy levels.

\subsection{Quantum optics}

We can also exploit the generality of the analysis in section 2 by addressing a less widely familiar case, the core mathematics in the field of quantum optics [21, 22]. To address the connection between the SHO and the electromagnetic field in free space, we again begin with the classical form of Hamiltonian. Specifically, the latter can be written as the volume integral of an energy density for the electromagnetic field $[2,13]$ as given by

$$
E=\frac{1}{2} \int_{V} \varepsilon_{0}\left(\mathbf{E}^{2}(\mathbf{r}, t)+c^{2} \mathbf{B}^{2}(\mathbf{r}, t)\right) \mathrm{d} V
$$

Here, the vector electric and magnetic fields $\mathbf{E}(\mathbf{r}, t)$ and $\mathbf{B}(\mathbf{r}, t)$, functions of the spatial position variables $\mathbf{r}=(x, y, z)$ and time $t$, can each be cast in the form of a sum over optical modes $a_{\mathbf{k}}^{(\lambda)}(t)$ of the potential vector $a(\mathbf{r}, t)$ (i.e. a mode expansion) satisfying Maxwell's equations within the Coulomb gauge [13]

$$
\begin{aligned}
& \mathbf{E}(\mathbf{r}, t)=\mathrm{i} c \sum_{\mathbf{k}, \lambda} k\left[\mathrm{e}^{(\lambda)}(\mathbf{k}) a_{\mathbf{k}}^{(\lambda)}(t) \mathrm{e}^{\mathrm{i} \mathbf{k} . \mathbf{r}}-\bar{e}^{(\lambda)}(\mathbf{k}) \bar{a}_{\mathbf{k}}^{(\lambda)}(t) \mathrm{e}^{-\mathrm{i} \mathbf{k} . \mathbf{r}}\right], \\
& \mathbf{B}(\mathbf{r}, t)=\mathrm{i} \sum_{\mathbf{k}, \lambda} k\left[\left(\hat{\mathbf{k}} \times \mathrm{e}^{(\lambda)}(\mathbf{k})\right) a_{\mathbf{k}}^{(\lambda)}(t) \mathrm{e}^{\mathrm{i} \mathbf{k} . \mathbf{r}}-\left(\hat{\mathbf{k}} \times \overline{\mathrm{e}}^{(\lambda)}(\mathbf{k})\right) \bar{a}_{\mathbf{k}}^{(\lambda)}(t) \mathrm{e}^{-\mathrm{i} \mathbf{k} . \mathbf{r}}\right],
\end{aligned}
$$

where a pair of orthogonal unit vectors, $\mathrm{e}^{(\lambda)}(\mathbf{k})$, serve to identify the polarization of the field and form a right-handed triad together with the wave-vector $\mathbf{k}$, i.e. $\left(\mathrm{e}^{(1)}(\mathbf{k}), \mathrm{e}^{(2)}(\mathbf{k}), \mathbf{k}\right)$. In 
passing, we note the parity character: the electric field is even in time, odd in space, whereas the magnetic field is odd in time, even in space.

It now follows that the energy density (29) is expressible as a sum of independent harmonic oscillators. On substituting the field expansions (30), (31) into (29) and performing the integration over space, the following compact expression can be obtained:

$$
H=2 V \varepsilon_{o} c^{2} \sum_{\mathbf{k}, \lambda} k^{2} a_{\mathbf{k}}^{(\lambda)} \bar{a}_{\mathbf{k}}^{(\lambda)}
$$

The field variable $a_{\mathbf{k}}^{(\lambda)}$ and its complex conjugate $\bar{a}_{\mathbf{k}}^{(\lambda)}$ can be written in terms of real and canonical conjugate variables, $q_{\mathbf{k}}^{(\lambda)}$ and $p_{\mathbf{k}}^{(\lambda)}$ :

$$
\begin{aligned}
& q_{\mathbf{k}}^{(\lambda)}=\left(\varepsilon_{0} V\right)^{1 / 2}\left(a_{\mathbf{k}}^{(\lambda)}+\bar{a}_{\mathbf{k}}^{(\lambda)}\right), \\
& p_{\mathbf{k}}^{(\lambda)}=-i c k\left(\varepsilon_{0} V\right)^{1 / 2}\left(a_{\mathbf{k}}^{(\lambda)}-\bar{a}_{\mathbf{k}}^{(\lambda)}\right) .
\end{aligned}
$$

Rewriting the energy density in terms of these new variables,

$$
H=\frac{1}{2} \sum_{\mathbf{k}, \lambda}\left(p_{\mathbf{k}}^{(\lambda)^{2}}+c^{2} k^{2} q_{\mathbf{k}}^{(\lambda)^{2}}\right)
$$

a simple harmonic form that exhibits the product $c k$ as the optical frequency. A more detailed account of the derivation can be found in $[2,13]$. So effectively, the energy density of the electromagnetic radiation has been decomposed into a set of SHOs, one for each mode $(\mathbf{k}, \lambda)$. The quantization of the electromagnetic field follows. As in section 3.1, operators $\hat{p}_{\mathbf{k}}^{(\lambda)}$ and $\hat{q}_{\mathbf{k}}^{(\lambda)}$ can be assigned to the variables $p_{\mathbf{k}}^{(\lambda)}$ and $q_{\mathbf{k}}^{(\lambda)}$. In consequence $a_{\mathbf{k}}^{(\lambda)}$ and its complex conjugate $\bar{a}_{\mathbf{k}}^{(\lambda)}$, also promoted to the quantum operator status, acquire the role of ladder operators

$$
\left.\begin{array}{l}
a_{\mathbf{k}}^{(\lambda)} \rightarrow\left(\hbar / 2 \varepsilon_{o} V c k\right)^{1 / 2} a_{\mathbf{k}}^{(\lambda)} \\
\bar{a}_{\mathbf{k}}^{(\lambda)} \rightarrow\left(\hbar / 2 \varepsilon_{o} V c k\right)^{1 / 2} a_{\mathbf{k}}^{(\lambda) \dagger}
\end{array}\right\},
$$

whose operation on number states follows the form of equations (15) and (16). The relation between each electromagnetic mode and a corresponding harmonic oscillator is the basis of quantum optics, where the commutation relation between the ladder operator $a_{\mathbf{k}}^{(\lambda)}$ and its Hermitian counterpart $a_{\mathbf{k}}^{(\lambda) \dagger}$ signifies the boson character of the radiation quanta [21]. Within the quantum description, the electromagnetic fields are recast in terms of these operators:

$$
\begin{aligned}
\mathbf{e}(\mathbf{r})=\mathrm{i} \sum_{\mathbf{k}, \lambda}\left(\frac{\hbar c k}{2 \varepsilon_{o} V}\right)^{1 / 2}\left[\mathrm{e}^{(\lambda)}(\mathbf{k}) a^{(\lambda)}(\mathbf{k}) \mathrm{e}^{\mathrm{i} \mathbf{k r}}-\overline{\mathrm{e}}^{(\lambda)}(\mathbf{k}) a^{\dagger(\lambda)}(\mathbf{k}) \mathrm{e}^{-\mathrm{i} \mathbf{k r}}\right] \\
\mathbf{b}\left(\mathbf{r}^{\prime}\right)=\nabla \times \mathbf{a}\left(\mathbf{r}^{\prime}\right)=\nabla \times \sum_{\mathbf{k}^{\prime}, \lambda^{\prime}}\left(\frac{\hbar}{2 \varepsilon_{o} c k^{\prime} V}\right)^{1 / 2} \\
\times\left[\mathrm{e}^{\left(\lambda^{\prime}\right)}\left(\mathbf{k}^{\prime}\right) a^{\left(\lambda^{\prime}\right)}\left(\mathbf{k}^{\prime}\right) \mathrm{e}^{\mathrm{i} \mathbf{k}^{\prime} \mathbf{r}^{\prime}}+\overline{\mathrm{e}}^{\left(\lambda^{\prime}\right)}\left(\mathbf{k}^{\prime}\right) a^{\dagger\left(\lambda^{\prime}\right)}\left(\mathbf{k}^{\prime}\right) \mathrm{e}^{-i \mathbf{k}^{\prime} \mathbf{r}^{\prime}}\right]
\end{aligned}
$$

which can be seen to obey the commutation rule $\left[e_{i}(\mathbf{r}), b_{j}\left(\mathbf{r}^{\prime}\right)\right]=\mathrm{i} \hbar \varepsilon_{o}^{-1} \varepsilon_{i j k} \nabla_{k} \delta\left(\mathbf{r}-\mathbf{r}^{\prime}\right)$.

The above analysis invites a brief reflection on the didactic conception of the photon as a particle. When viewed as such, it appears natural that light energy of a given optical frequency will be manifest as multiples of a single photon energy. However, when the number of photons in a given radiation mode is properly conceived as a quantum number, it becomes evident that such energy additivity is not a feature that can in general be taken for granted. The energy difference between states with $n=1$ and $n=3$ photons is of course twice the difference between the $n=1$ and $n=2$ states, but it is equally obvious that this rule is not applicable to, say, the ratio of intervals between the energy levels of a hydrogen atom. It is 
also worth observing that, since the above analysis allows the zero-point energy for each mode of oscillation also to be deduced, its application in a quantum optics context affords a very natural introduction to the subject of vacuum fluctuations [23] - material that is more than enough for another lecture.

\section{Conclusion}

It proves highly instructive to tackle simple harmonic oscillation from the starting point of observations on the structure of the associated quantum energy levels. The familiar evenly spaced stacking of these levels is a feature that, even alone, can furnish astonishingly rich information on the mathematical structure of the quantum Hamiltonian-one whose relationship to a classical energy expression then allows further inferences to be drawn in the case of specific physical systems. In section 2, it has been shown possible to deduce from the observed structure of the quantum energy levels that the Hamiltonian for SHO must accommodate terms that are quadratic in two dynamical variables with non-commuting quantum operators. As demonstrated in section 3, these variables are none other than position and momentum in a system of mechanical oscillation, with a similar role being played by the electric and magnetic fields in the case of optical radiation.

Although the inverse inference is well known, it is particularly striking that the very existence of photons manifestly owes its origins to an energy structure of simple harmonic form. An assumed familiarity with this linkage, widely taken for granted, can mask the power and reversibility of the underlying logic. It is also notable that the facility to comprehend photons in particulate terms would be insupportable, when applied to many-photon systems, without the equidistant placement of successive quantum energy levels. This is consistent with the received wisdom that it is unfeasible for photons to directly interact with each other [24]although there is of course a variety of means whereby they interact, through an engagement with matter.

Once recognized as a singular feature of a simple harmonic dynamical structure, the energy additivity of electromagnetic quanta presents an unusual perspective to the underlying physics, and we believe there are significant pedagogical advantages to be accrued from this approach. Another, more philosophical advantage is to develop theory from physical arguments that are squarely based on measurement, a 'top-down' approach faithfully in keeping with the very spirit of quantum mechanics [25]. In developing the quantum theory of light, the most common approach is to begin with Maxwell's equations and work upwards from there, but despite a well-justified confidence in such a foundation its basis is arguably more remote from experimental observations.

\section{Acknowledgments}

The authors gladly thank Professor M Babiker and an anonymous referee for helpful comments on the analysis. This work is supported by a grant from the Engineering and Physical Sciences Research Council.

\section{References}

[1] Cohen-Tannoudji C, Dupont-Roc J and Grynberg G 2004 Photons and Atoms: Introduction to Quantum Electrodynamics (Weinheim: Wiley)

[2] Mandel L and Wolf E 1995 Optical Coherence and Quantum Optics (Cambridge: Cambridge University Press)

[3] Robinett R W 2006 Quantum Mechanics: Classical Results, Modern Systems, and Visualized Examples 2nd edn (Oxford: Oxford University Press) 
[4] Sakurai J J 1994 Modern Quantum Mechanics ed S F Tuan, revised edition (Wokingham: Addison-Wesley)

[5] Mahan G D 2009 Quantum Mechanics in a Nutshell (Princeton, NJ: Princeton University Press)

[6] Calogero F 1969 Solution of a three-body problem in one dimension J. Math. Phys. 10 2191-6

[7] Calogero F 1996 Solution of the one-dimensional N-body problems with quadratic and/or inversely quadratic pair potentials J. Math. Phys. 37 3646-6 (erratum)

[8] Sutherland B 1971 Quantum many-body problem in one dimension: ground state J. Math. Phys 12 246-50

[9] Sutherland B 1971 Quantum many-body problem in one dimension: thermodynamics J. Math. Phys. 12 251-6

[10] Calogero F 1971 Solution of the one-dimensional N-body problems with quadratic and/or inversely quadratic pair potentials J. Math. Phys. 12 419-36

[11] Gurappa N and Panigrahi P K 1999 Equivalence of the Calogero-Sutherland model to free harmonic oscillators Phys. Rev. B 59 R2490

[12] Messiah A, Temmer G M and Potter J 1999 Quantum Mechanics (Mineola, NY: Dover)

[13] Craig D P and Thirunamachandran T 1998 Molecular Quantum Electrodynamics: An Introduction to RadiationMolecule Interactions (Mineola, NY: Dover)

[14] Cohen-Tannoudji C, Diu B and Laloe F 1977 Quantum Mechanics 2nd edn (New York: Wiley)

[15] Ballentine L E 2003 Quantum Mechanics: A Modern Development (Singapore: World Scientific)

[16] Schwinger J and Englert B-G 2001 Quantum Mechanics: Symbolism of Atomic Measurements (Berlin: Springer)

[17] Bowman G E 2008 Essential Quantum Mechanics (Oxford: Oxford University Press) Chapter 7

[18] Shankar R 1994 Principles of Quantum Mechanics (New York: Springer) Chapter 9

[19] Bohm A 2001 Quantum Mechanics: Foundations and Applications (New York: Springer)

[20] Landau L D and Lifshitz E M 1999 Mechanics (Oxford: Butterworth-Heinemann)

[21] Loudon R 2000 The Quantum Theory of Light (Oxford: Oxford University Press)

[22] Gerry C C and Knight P 2005 Introductory Quantum Optics (New York: Cambridge University Press)

[23] Scully M O and Zubairy M S 1997 Quantum Optics (Cambridge: Cambridge University Press)

[24] Dirac P A M 1958 The Principles of Quantum Mechanics 4th edn (Oxford: Oxford University Press) p 9

[25] Baggott J E 2004 Beyond Measure: Modern Physics, Philosophy, and the Meaning of Quantum Theory (Oxford: Oxford University Press) Chapter 5 